\title{
Vascular Invasion and Metastasis is Predictive of Outcome in Barcelona Clinic Liver Cancer Stage C Hepatocellular Carcinoma
}

\author{
Ali A. Mokdad, MD, MS ; ; Amit G. Singal, MD, MS' ; Jorge A. Marrero, MD' ${ }^{\text {b }}$ Hao Zhu, MDc; and \\ Adam C. Yopp, MDa
}

\begin{abstract}
Background: Patients with Barcelona Clinic Liver Cancer (BCLC) stage C hepatocellular carcinoma (HCC) have variable long-term outcomes. Better delineation of prognosis is important for clinical trial enrollment and clinical practice in an era of precision medicine. We hypothesized that stratification of patients with BCLC stage C HCC by presence of vascular invasion and/or metastasis improves prognostic discrimination. Methods: Using a prospectively maintained database, we identified 234 patients diagnosed with BCLC stage C HCC between 2005 and 2015. Patients were stratified into 3 groups based on tumor characteristics: (1) vascular invasion alone, (2) metastasis alone, and (3) vascular invasion and metastasis. Overall survival (OS) was compared using a Cox model. A subgroup analysis was performed based on extent of vascular invasion and site of metastasis. Results: The cohort comprised 123 patients $(53 \%)$ with vascular invasion alone, 34 (15\%) with metastasis alone, and $77(33 \%)$ with both vascular invasion and metastasis. Median survival was 5.7, 3.9, and 3.0 months, respectively $(P<.01)$. Patients with vascular invasion or metastasis alone had significantly better survival compared with those with vascular invasion and metastasis (adjusted hazard ratio [HR],0.68; $95 \% \mathrm{Cl}, 0.49-0.94$, and $\mathrm{HR}, 0.61 ; 95 \% \mathrm{Cl}, 0.39-0.96$, respectively). Compared with tumoral invasion of branch portal veins, involvement of the main portal vein was associated with worse survival (HR, 2.13; 95\% Cl, 1.29-3.49). OS did not differ by site of metastasis. Conclusions: Stratification of patients within the BCLC stage C staging subgroup by vascular invasion and presence of metastasis further discriminates patient prognosis. This substratification may have implications for therapy and more accurate prognostic features.
\end{abstract}

\section{Background}

The incidence and mortality of hepatocellular carcinoma (HCC) have increased 2-fold during the past 2 decades, and it is currently the fastest growing cause of cancerrelated death in the United States. ${ }^{1}$ The 5 -year survival rate for HCC remains below 20\% in the United States. ${ }^{2}$ Poor HCC outcomes are largely attributed to low rates of HCC surveillance and high rates of late stage diagnosis. 3,4

Accurate HCC staging is important for patient prognostication, treatment delivery, and standardiza-

From a Department of Surgery, Division of Surgical Oncology; ${ }^{\mathrm{b} D e p a r t m e n t}$ of Medicine, Division of Digestive and Liver Diseases; and 'Children's Research Institute, Departments of Pediatrics and Internal Medicine, Center for Regenerative Science and Medicine, University of Texas Southwestern Medical Center, Dallas, Texas.

Submitted June 16, 2016; accepted for publication October 13, 2016.

The authors have disclosed that they have no financial interests, arrangements, affiliations, or commercial interests with the manufacturers of any products discussed in this article or their competitors. tion of inclusion criteria for patients enrolled in clinical trials. Although there is no universally accepted staging system, the Barcelona Clinic Liver Cancer (BCLC) staging system has been adopted by both the American Association for the Study of Liver Diseases (AASLD) and the European Association for the Study of the Liver. ${ }^{5,6}$ The BCLC classification stratifies patients according to tumor stage, liver function, and ECOG performance status (PS) into 4 categories ( 0 and A, early; B, intermediate; C, advanced; D, end-stage). ${ }^{7,8}$ The BCLC

Author contributions: Conceptualization and methodology: Mokdad, Singal, Marrero, Zhu, Yopp. Formal analysis: Mokdad. Drafted manuscript: Mokdad, Yopp. Manuscript review and editing: Mokdad, Singal, Marrero, Zhu, Yopp.

Correspondence: Ali A. Mokdad, MD, MS, Department of Surgery, Division of Surgical Oncology, University of Texas Southwestern Medical Center, 5323 Harry Hines Boulevard, Dallas, TX 75390.

E-mail: ali.elmokdad@utsouthwestern.edu 
Mokdad et al

classification has been externally validated in Western and Asian populations and demonstrates superior prognostic discrimination compared with other HCC staging systems. ${ }^{9,10}$

Although prognosis and treatment are fairly uniform at the ends of the BCLC classification (BCLC stages $\mathrm{A}$ and $\mathrm{D})$, heterogeneity exists within BCLC stages $B$ and $C$ due to variable tumor characteristics and degrees of liver dysfunction. ${ }^{11,12}$ In addition, median survival for patients with BCLC stage C HCC ranges from 3 to 10 months, further underscoring the nonhomogenous nature of this group. ${ }^{10-14}$ In the era of precision medicine, this large variability in survival can serve as a source of consternation for patients with newly diagnosed HCC. However, in the absence of routine biopsies for HCC, we are forced to depend on readily available clinical characteristics for improved prognostication.

This study explored whether patients with BCLC stage C HCC could be further stratified by tumor characteristics to improve HCC prognostication and facilitate appropriate treatment decisions.

\section{Methods}

\section{Study Population}

Using a prospectively maintained database of patients with HCC seen at Parkland Health \& Hospital System (PHHS) and the University of Texas Southwestern (UTSW) Medical Center, we identified patients diagnosed with BCLC stage C HCC between January 2005 and June 2015. PHHS is an integrated safetynet health system and UTSW is an NCI-designated tertiary care referral center. One multidisciplinary team attends to the care of patients with $\mathrm{HCC}$ at both institutions, as described elsewhere. ${ }^{15}$

The diagnosis of HCC was based on AASLD criteria, ${ }^{5}$ and all cases were adjudicated by one of two authors (A.G.S. or A.C.Y.). Patients were classified as BCLC stage $\mathrm{C}$ based on the presence of vascular invasion or metastasis, including spread to lymph nodes and/or distant organs. Vascular invasion was distinguished from bland thrombus by the presence of arterial enhancement, portal venous expansion, or development of new thrombus directly contiguous with the tumor. Metastatic lymph nodes were diagnosed by histologic confirmation or, predominantly, by radiographic evidence of enlarged nodes $(>1.5 \mathrm{~cm})$ outside of the periportal region, enlarged nodes $(>3 \mathrm{~cm})$ within the periportal region, or enlarging lymph nodes on serial imaging. Metastases to distant organs was diagnosed by histologic confirmation in most cases but could also be diagnosed by radiographic evidence of an enhancing mass $>1.0 \mathrm{~cm}$ in distant organs. Patients with Child-Pugh class $\mathrm{C}$ cirrhosis or ECOG PS $>2$ were classified as BCLC stage D and excluded from this study. This study was approved by the Institutional Review Boards at both institutions.

\section{Data Collection}

Patient demographic data, clinical history, and longterm follow-up data were abstracted from patients' electronic medical records, as described elsewhere. ${ }^{16}$ Relevant medical history, baseline biochemical laboratory results, tumor characteristics at presentation, and first course of treatment were collected. Model for End-Stage Liver Disease (MELD) and albuminbilirubin (ALBI) scores were calculated. ${ }^{17,18}$ An infiltrative pattern was defined as an ill-defined diffuse liver mass on a background of cirrhosis. Metastasis comprised nodal spread or spread to distant organs. Vascular invasion was divided into 4 categories: branch portal vein, left/right or main portal trunk, hepatic vein, and a combination of portal and hepatic vein involvement. Treatment variables included surgical resection, local ablative therapies, transarterial chemoembolization (TACE), transarterial radioembolization (TARE), and systemic therapy, including sorafenib or investigational therapies as part of a clinical trial. We differentiated treatment preceding and following 2008 to reflect introduction of sorafenib for the treatment of advanced HCC. Treatment decisions for all patients were based on discussions and consensus recommendations at a multidisciplinary liver tumor conference, as described elsewhere. ${ }^{15}$

\section{Statistical Analysis}

We divided patients into 3 categories: (1) vascular invasion alone, (2) metastasis alone, and (3) both vascular invasion and metastasis. We compared baseline patient, tumor, and treatment variables using analysis of variance and chi-square test for continuous and categorical variables, respectively. We constructed Kaplan-Meier curves and compared overall survival (OS) using the log-rank test. We used Cox proportional hazard regression model to evaluate adjusted differences in survival. Clinically relevant covariates 
BCLC Stage C Hepatocellular Carcinoma

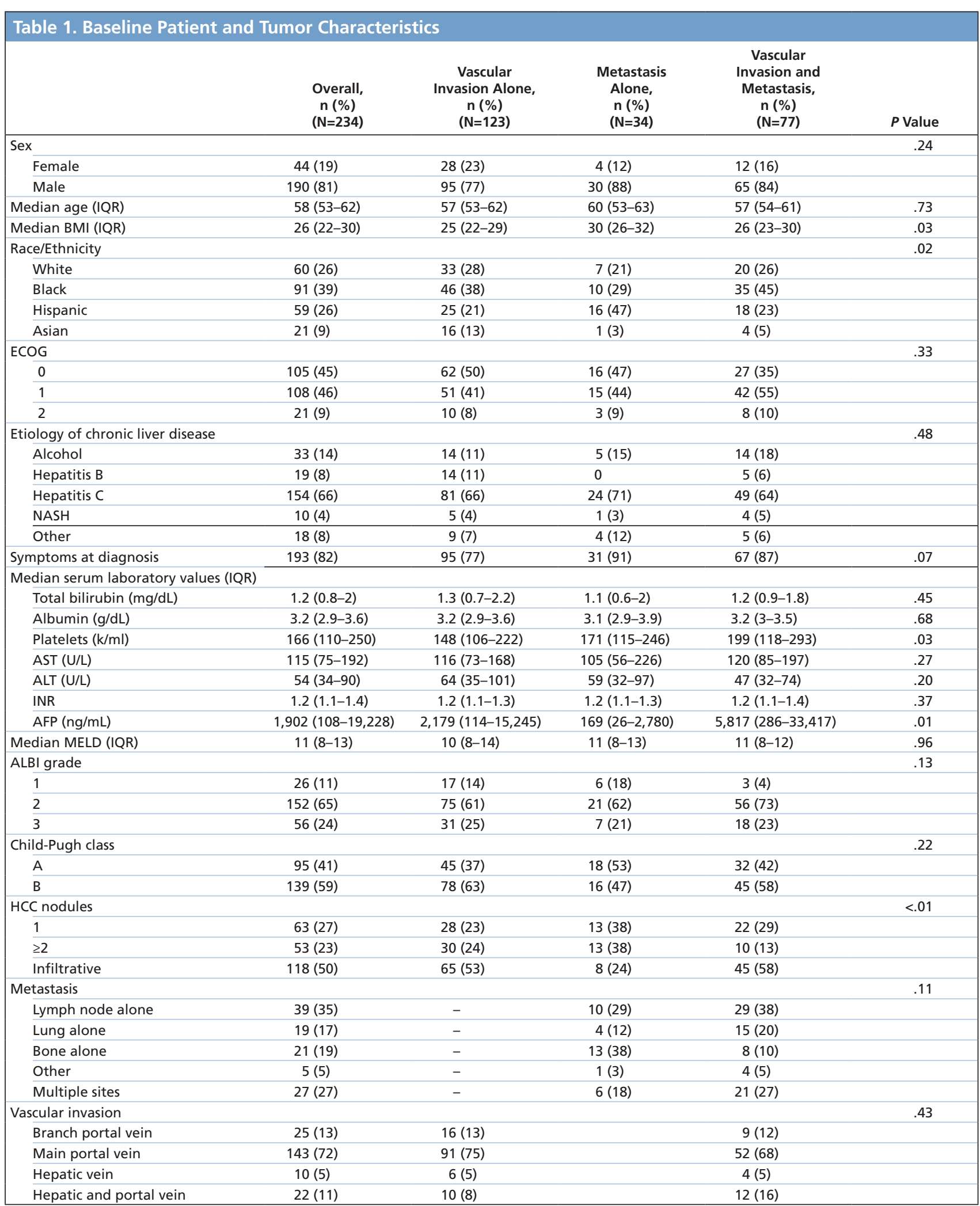

Abbreviations: AFP, alpha-fetoprotein; ALBI, albumin-bilirubin; ALT, alanine transaminase; AST, aspartate transaminase; BMI, body mass index; HCC, hepatocellular carcinoma; INR, international normalized ratio; IQR, interquartile ratio; MELD, Model for End-Stage Liver Disease; NASH, nonalcoholic steatohepatitis. 
Mokdad et al

\begin{tabular}{|c|c|c|c|c|c|}
\hline & $\begin{array}{l}\text { Overall, } n(\%) \\
\quad(N=234)\end{array}$ & $\begin{array}{c}\text { Vascular Invasion } \\
\text { Alone, } \mathrm{n}(\%) \\
(\mathrm{N}=123)\end{array}$ & $\begin{array}{c}\text { Metastasis } \\
\text { Alone, } \mathrm{n}(\%) \\
(\mathrm{N}=34)\end{array}$ & $\begin{array}{c}\text { Vascular Invasion } \\
\text { and Metastasis, } \\
n(\%) \\
(\mathrm{N}=77)\end{array}$ & $P$ Value \\
\hline Systemic chemotherapy & & & & & .12 \\
\hline 2005-2008 & $7(3)$ & $4(3)$ & $3(9)$ & 0 & \\
\hline 2009-2015 & $71(31)$ & $33(28)$ & $11(33)$ & $27(36)$ & \\
\hline Sorafenib & $74(33)$ & $35(30)$ & $13(39)$ & $26(35)$ & .60 \\
\hline Transarterial chemoembolization & $14(6)$ & $9(8)$ & $5(15)$ & 0 & $<.01$ \\
\hline Yttrium-90 radioembolization & $4(2)$ & $4(3)$ & 0 & 0 & .21 \\
\hline Radiation (to liver) & $3(1)$ & $3(3)$ & 0 & 0 & .40 \\
\hline Radiation (to metastasis) & $8(4)$ & 0 & $6(18)$ & $2(3)$ & $<.01$ \\
\hline Any treatment & $99(44)$ & $52(44)$ & $19(56)$ & $28(37)$ & .18 \\
\hline
\end{tabular}

(ECOG PS, Child-Pugh class, and treatment) and those with $P \leq .2$ on univariate Wald test were evaluated in the multivariable model. The final model was constructed using a forward selection procedure. The proportional hazard assumption was evaluated and deemed reasonable based on graphic examination of scaled Schoenfeld residuals. We performed subgroup analyses to evaluate survival by site of metastasis and degree of vascular invasion. All tests were 2-sided and performed at a 5\% significance level. Statistical analysis was performed using STATA 14 (StataCorp LP, College Station, TX).

\section{Results}

\section{Baseline Characteristics}

Of 234 patients with BCLC stage C HCC at initial presentation, 123 (53\%) had vascular invasion alone, 34 (15\%) had metastasis alone, and 77 (33\%) had both vascular invasion and metastatic disease. Patient age $(P=.73)$ and sex $(P=.24)$ were similar among all groups (Table 1 ). Patients in the metastasis alone group were more likely to be Hispanic, whereas those with vascular invasion, with or without concomitant metastatic disease, were more likely to be black $(P=.02)$. Hepatitis $C$ was the most common cause of chronic liver disease. Laboratory data were notable for higher alpha fetoprotein levels among those with vascular invasion, with or without metastasis, compared with patients with metastasis alone $(P=.01)$. There was no difference in liver function, which was estimated using MELD score, ALBI grade, or Child-Pugh class, among the groups. $\mathrm{Pa}$ tients with metastatic disease alone were more likely to have unifocal liver tumors. Infiltrative HCC was more prevalent in patients with vascular invasion with $(58 \%)$ or without $(53 \%)$ metastasis compared with metastasis alone $(24 \%)(P<.01)$.

Receipt of any treatment did not significantly differ among the 3 groups $(P=.18)$ (Table 2$)$. Reasons for no treatment included clinical deterioration before initiating treatment $(31 \%)$, poor liver function $(29 \%)$, and patient refusal (12\%). Treatment with sorafenib was the most common treatment, ranging between $30 \%$ and $39 \%(P=.60)$. In each group, $10 \%$ to $15 \%$ of patients received locoregional therapy, namely TACE, yttrium-90 $\left({ }^{90} \mathrm{Y}\right)$ radioembolization (TARE), or stereotactic body radiation therapy. These therapies were offered more commonly to patients with vascular invasion alone or metastasis alone than to patients with vascular invasion and metastasis (14\% and $15 \%$ vs $0 \%$, respectively; $\mathrm{P}<.01)$. Patients with branch portal venous thrombus were more likely than those with main portal venous thrombus to receive either TACE or TARE ( $16 \%$ vs $5 \% ; P=.04)$.

\section{Overall Survival}

During a median follow-up of 3.0 months (average, 5.2 months), a total of 203 patients (87\%) died. Overall median survival was 3.7 months and survival rates at 6,12 , and 24 months were $32 \%, 12 \%$, and $5 \%$, respectively. Patients with both vascular invasion and metastasis had worse survival than those with vascular invasion alone and metastasis alone (median survival, 3.0 vs 3.9 and 5.7 months, respectively; $P<.01$; Figure 1 ). Table 3 details median OS according to Child-Pugh class. On multivari- 
BCLC Stage C Hepatocellular Carcinoma

\begin{tabular}{|c|c|c|}
\hline & $\begin{array}{c}\text { Child-Pugh } \\
\text { Class A } \\
(95 \% \mathrm{Cl})\end{array}$ & $\begin{array}{c}\text { Child-Pugh } \\
\text { Class B } \\
(95 \% \text { Cl) }\end{array}$ \\
\hline \multicolumn{3}{|l|}{ BCLC stage $\mathrm{C}$ group } \\
\hline $\begin{array}{l}\text { Vascular } \\
\text { invasion alone }\end{array}$ & $6.7(5.2-10.3)$ & $2.5(1.9-2.8)$ \\
\hline Metastasis alone & $7.5(5.6-9.3)$ & $3.8(1.0-5.7)$ \\
\hline $\begin{array}{l}\text { Vascular invasion } \\
\text { and metastasis }\end{array}$ & $3.8(2.8-6.1)$ & $1.9(1.3-3.0)$ \\
\hline
\end{tabular}

Abbreviation: BCLC, Barcelona Clinic Liver Cancer.

able analysis, patients with both vascular invasion and metastasis had an adjusted hazard ratio (HR) of 1.46 (95\% CI, 1.06-2.02) compared with those with vascular invasion alone, and an HR of 1.65 (95\% CI, 1.04-2.59) compared with those with metastasis alone (Table 4). Patients with metastasis alone had similar OS as those with vascular invasion alone (HR, 0.89; 95\% CI, 0.56-1.38).

\section{Vascular Invasion Subgroup}

Among patients with vascular invasion, with and without concomitant metastatic disease, most presented with portal vein invasion: 25 patients $(13 \%)$ had branch portal vein invasion and 143 (72\%) had main portal vein invasion. The remaining $16 \%$ presented with hepatic vein invasion alone $(5 \%)$ or a combination of portal vein and hepatic vein involvement (11\%) (Table 1). Patients with branch portal vein involvement were most likely to receive treatment, whereas those with both hepatic vein and portal vein invasion were least likely to receive treatment ( $50 \%$ vs $14 \% ; P=.05$ ). Patients with main portal vein invasion had worse survival compared with those with branch portal vein invasion (median survival, 3.2 vs 4.7 months; HR, 2.13; 95\% CI, 1.29-3.49; Figure 2 and Table 5), although median OS was shortest in patients with both portal vein and hepatic vein invasion (2.6 months).

\section{Metastasis Subgroup}

More than half of patients with metastasis, irrespective of tumoral vascular invasion, had lymph node involvement $(54 \%)$, and this was the only site of metastasis in $35 \%$ of patients (Table 1 ). The most common site of metastasis in the metastasis alone group was bone $(38 \%)$. In the group with vascular invasion and metastasis, lymph node alone (38\%) and disseminated metastases $(27 \%)$ were the most common sites. The site of metastasis was associated with differences in OS (Table 5). Patients with bone metastasis alone had better survival compared with metastases to other sites (median survival, 4.2 vs 3.0 months; $P=.03$ ). Differences in survival by site of metastases dissipated, however, after adjustment for liver function, intrahepatic HCC burden, and treatment variables (Table 5).

\section{Discussion}

Overall prognosis and treatment decision-making in patients with HCC are incumbent on appropriate patient stratification. Although BCLC-based stratification adequately differentiates patients with favorable prognosis (BCLC stage $\mathrm{A}$ ) from those with terminal disease (BCLC stage D), most patients with HCC occupy the middle of the spectrum (BCLC stages B and $\mathrm{C}$ ), where treatment and overall prognosis remain less well defined. ${ }^{7,8,11}$ In this study, we showed that presentation with both vascular invasion and metastatic disease was associated with significantly worse survival than vascular invasion or metastasis alone. Our findings suggest that variability in prognosis among patients with BCLC stage C HCC is accounted for, at least partly, by tumoral vascular invasion and metastasis-based groupings.

Treatment algorithms based on BCLC staging do not distinguish between patients with different vascular invasion patterns, and sorafenib is recommended for patients with any degree of vascular invasion. ${ }^{5,6}$

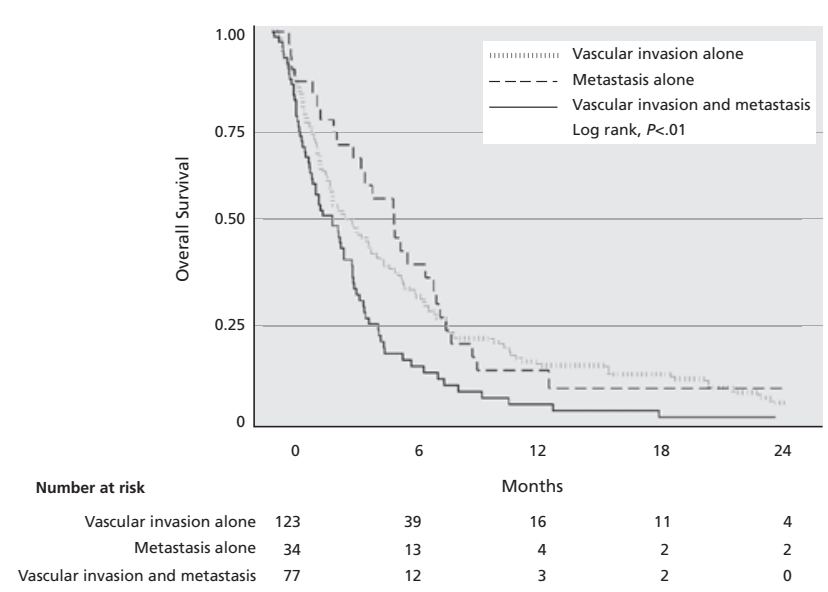

Figure 1. Overall survival by Barcelona Clinic Liver Cancer stage subgroup. 
Our findings and data from other studies similarly observed a more favorable prognosis when tumoral involvement was limited to small caliber downstream branches of the portal system. ${ }^{19-22}$ In addition to having a better prognosis, select patients with branch tumor thrombus may be successfully treated with curative therapies, such as surgical resection, or locoregional therapies, such as TACE or TARE. ${ }^{14,23,24}$ It is becoming increasingly clear that all patients with vascular invasion do not constitute a homogenous group, and that treatment decisions should be tailored based on the degree of vascular involvement.

In contrast, we did not find any differences in survival according to site of metastasis. After accounting for liver function and intrahepatic tumor characteristics, differences in survival dissipated. Although some studies have reported improved survival in patients with metastasis to lymph nodes compared with distant organs, ${ }^{19,25}$ several other cohorts, including those in our study, did not demonstrate a difference. ${ }^{26}$ Our finding conforms to the notion that prognosis in patients with metastatic HCC is largely driven by the intrahepatic lesion character-

\begin{tabular}{|c|c|c|c|c|}
\hline & Adjusted HR & $P$ Value & $95 \% \mathrm{Cl}$ & $\begin{array}{c}\text { Median } \\
\text { OS } \\
(95 \% \mathrm{Cl})\end{array}$ \\
\hline \multicolumn{5}{|l|}{ BCLC stage $C$ group } \\
\hline $\begin{array}{l}\text { Vascular invasion } \\
\text { alone }\end{array}$ & Ref & & & $\begin{array}{c}3.9 \\
(2.7-5.2)\end{array}$ \\
\hline Metastasis alone & 0.89 & .60 & $0.56-1.38$ & $\begin{array}{c}5.7 \\
(3.8-7.6)\end{array}$ \\
\hline $\begin{array}{l}\text { Vascular invasion } \\
\text { and metastasis }\end{array}$ & 1.46 & .02 & $1.06-2.02$ & $\begin{array}{c}3.0 \\
(1.9-3.7)\end{array}$ \\
\hline \multicolumn{5}{|c|}{ Cancer-related symptoms } \\
\hline Absent & Ref & & & $\begin{array}{c}7.1 \\
(3.4-11.6)\end{array}$ \\
\hline Present & 2.0 & $<.01$ & $1.30-3.04$ & $\begin{array}{c}3.2 \\
(2.7-3.9)\end{array}$ \\
\hline \multicolumn{5}{|l|}{ Child-Pugh class } \\
\hline A & Ref & & & $\begin{array}{c}6.0 \\
(5.0-7.3)\end{array}$ \\
\hline B & 1.72 & $<.01$ & $1.27-2.34$ & $\begin{array}{c}2.2 \\
(2.0-2.8)\end{array}$ \\
\hline \multicolumn{5}{|l|}{ Treatment } \\
\hline No & Ref & & & $\begin{array}{c}2.0 \\
(1.6-2.3)\end{array}$ \\
\hline Yes & 0.32 & $<.01$ & $0.24-0.44$ & $\begin{array}{c}6.1 \\
(5.2-7.3)\end{array}$ \\
\hline
\end{tabular}

Abbreviations: BCLC, Barcelona Clinic Liver Cancer; HR, hazard ratio; OS, overall survival.

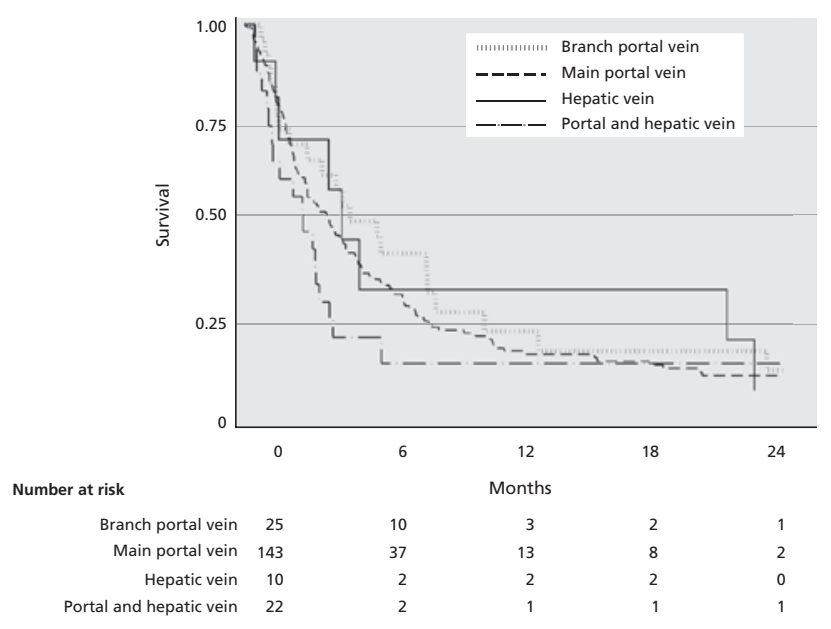

Figure 2. Overall survival by degree of vascular invasion.

istics and liver function, with most patients with metastatic HCC dying of progression of intrahepatic disease and/or liver failure. ${ }^{27-30}$ This finding has important ramifications for treatment, because current guidelines recommend systemic therapy including sorafenib for metastatic disease. Locoregional treatment of the intrahepatic tumor burden combined with systemic therapy may be more efficacious. This more aggressive approach has been advocated by the Hong Kong Liver Cancer (HKLC) staging system in patients primarily with hepatitis B virus (HBV)-related HCC. ${ }^{21}$ The HKLC system, however, has not been validated in patients with non-HBV-related HCC. Furthermore, more aggressive locoregional therapies, including TACE or TARE, have not been compared with sorafenib alone or in conjunction with sorafenib in patients with BCLC stage C HCC. Currently, multiple clinical trials are underway comparing locoregional therapies including TARE with sorafenib in the treatment of patients with BCLC stage $\mathrm{C} \mathrm{HCC}$ with malignant venous thrombus and without evidence of extrahepatic disease (ClinicalTrials.gov identifiers: NCT01482442 and NCT00712790). The results of these trials will allow further delineation of appropriate treatments for BCLC stage C HCC based on more discriminatory clinicopathologic factors instead of a "one size fits all" treatment approach.

Finally, we found that patients with a combination of vascular invasion and metastasis had significantly worse survival than those with either vascular invasion or metastasis alone. A similar finding was 
BCLC Stage C Hepatocellular Carcinoma

noted in an Asian cohort in which patients with main portal vein invasion and distant metastasis had worse survival compared with patients with either tumor characteristic alone (2.3 vs 4.9-5.7 months). ${ }^{19}$ The extremely poor prognosis in this subgroup of patients mirrors that of patients with BCLC stage D HCC and raises the question of whether any benefit is derived from locoregional or systemic therapy. Even in the subgroup of patients with Child-Pugh A cirrhosis and ECOG PS of 0 or 1 the median survival was 3.8 months. In the SHARP trial, a subgroup analysis of patients with vascular invasion and/or metastatic disease derived benefit from sorafenib; however, an analysis was not performed among those with both characteristics simulateneously. ${ }^{31}$ It is possible that these patients may be best treated with best supportive care, because further data are needed to evaluate the effectiveness and cost-effectiveness of therapy in this subgroup.

Notably, OS in this cohort of patients with BCLC stage C HCC was worse than what would have been expected based on findings from the SHARP trial. Still, it is unlikely that OS in this cohort was an outlier. Rather, it represents the reality of prognosis in patients with BCLC stage C HCC in real-life practice outside the "ideal" settings of a clinical trial. Unlike the SHARP trial, nearly 60\% of our patients had Child-Pugh class B cirrhosis and $82 \%$ were symptomatic at the time of diagnosis.

Findings from this study were interpreted in light of the following limitations. First, unmeasured potential confounders not accounted for in the regression models secondary to the nonrandomized study design may have biased the comparisons of OS. Second, inferences related to OS based on the site of metastasis were prone to type II error (failing to demonstrate a difference when one truly exists) due to a small sample size. Although the goal of our study was to move toward more personalized prognostication for patients with BCLC stage C HCC, our ability to

\begin{tabular}{|c|c|c|}
\hline & $\begin{array}{l}\text { Hazard Ratioa } \\
(95 \% \mathrm{Cl})\end{array}$ & $\begin{array}{l}\text { Median Survival } \\
\quad(95 \% \mathrm{Cl})\end{array}$ \\
\hline \multicolumn{3}{|l|}{ Vascular invasion } \\
\hline $\begin{array}{l}\text { Branch portal } \\
\text { vein }\end{array}$ & Ref & $4.7(1.5-8.1)$ \\
\hline $\begin{array}{l}\text { Main portal } \\
\text { vein }\end{array}$ & $2.13(1.29-3.49)$ & $3.3(2.5-4.2)$ \\
\hline Hepatic vein & $2.14(0.89-5.15)$ & $4.3(0.4-21.5)$ \\
\hline $\begin{array}{l}\text { Hepatic and } \\
\text { portal vein }\end{array}$ & $1.99(1.04-3.80)$ & $2.6(1.0-3.2)$ \\
\hline \multicolumn{3}{|l|}{ Metastases } \\
\hline $\begin{array}{l}\text { Lymph node } \\
\text { alone }\end{array}$ & Ref & $3.9(2.0-5.0)$ \\
\hline Lung alone & $1.15(0.64-2.07)$ & $2.8(1.2-5.2)$ \\
\hline Bone alone & $0.74(0.38-1.43)$ & $4.2(3.2-9.3)$ \\
\hline Other & $1.22(0.46-3.26)$ & 1.7 (0.07-ND) \\
\hline Multiple sites & $0.64(0.36-1.15)$ & $3.1(1.5-3.8)$ \\
\hline
\end{tabular}

Abreviation: ND, not defined.

${ }^{a}$ Adjusted hazard ratio based on the final Cox proportional hazard regression model that included site of metastasis, Child-Pugh class, presence of metastasis, and treatment.

do so is limited by absence of tumor genetic and pathology information given the lack of HCC biopsies in clinical practice.

\section{Conclusions}

Stratification of patients with BCLC stage C HCC by tumoral vascular invasion and metastasis further delineates patient prognosis. Patients with vascular invasion and concomitant metastasis have the worst survival, whereas patients with limited branch portal vein involvement have a distinctly favorable prognosis. Moving forward, these data should be used for determining prognosis and making treatment decisions for patients with BCLC stage C HCC and for stratifying patient selection in future clinical trials.

\section{References}

1. Global Burden of Disease Cancer Collaboration. The global burden of cancer 2013. JAMA Oncol. 2015;1:505-527.

2. SEER Stat Fact Sheets: Cancer of the Liver and Intrahepatic Bile Duct. Available at: https://seer.cancer.gov/statfacts/html/livibd.html. Accessed January 4, 2017.

3. Tan D, Yopp A, Beg MS, et al. Meta-analysis: underutilisation and disparities of treatment among patients with hepatocellular carcinoma in the United States. Aliment Pharmacol Ther 2013;38:703-712.

4. Singal AG, Yopp A, S. Skinner C, et al. Utilization of hepatocellular carcinoma surveillance among American patients: a systematic review. J Gen Intern Med 2012;27:861-867.

5. Bruix J, Sherman M. Management of hepatocellular carcinoma: an update. Hepatology 2011;53:1020-1022.

6. European Association for the Study of the Liver, European Organisation for Research and Treatment of Cancer. EASL-EORTC clinical practice guidelines: management of hepatocellular carcinoma. J Hepatol 2012;56:908-943. 
7. Llovet JM, Bustamante J, Castells A, et al. Natural history of untreated nonsurgical hepatocellular carcinoma: rationale for the design and evaluation of therapeutic trials. Hepatology 1999;29:62-67.

8. Llovet JM, Brú C, Bruix J. Prognosis of hepatocellular carcinoma: the BCLC staging classification. Semin Liver Dis 1999;19:329-338.

9. Marrero JA, Fontana RJ, Barrat A, et al. Prognosis of hepatocellular carcinoma: comparison of 7 staging systems in an American cohort. Hepatology 2005;41:707-716.

10. Maida M, Orlando E, Cammà C, Cabibbo G. Staging systems of hepatocellular carcinoma: a review of literature. World J Gastroenterol 2014;20:4141-4150.

11. Forner A, Reig ME, de Lope CR, Bruix J. Current strategy for staging and treatment: the BCLC update and future prospects. Semin Liver Dis 2010;30:61-74.

12. Forner A, Gilabert M, Bruix J, Raoul JL. Treatment of intermediate-stage hepatocellular carcinoma. Nat Rev Clin Oncol 2014;11:525-535.

13. Park JW, Chen M, Colombo M, et al. Global patterns of hepatocellular carcinoma management from diagnosis to death: the BRIDGE study. Liver Int 2015;35:2155-2166.

14. Torzilli G, Belghiti J, Kokudo N, et al. A snapshot of the effective indications and results of surgery for hepatocellular carcinoma in tertiary referral centers: is it adherent to the EASL/AASLD recommendations? an observational study of the HCC East-West study group. Ann Surg 2013;257:929-937.

15. Yopp AC, Mansour JC, Beg MS, et al. Establishment of a multidisciplinary hepatocellular carcinoma clinic is associated with improved clinical outcome. Ann Surg Oncol 2014;21:1287-1295.

16. Yopp AC, Mokdad A, Zhu H, et al. Infiltrative hepatocellular carcinoma: natural history and comparison with multifocal, nodular hepatocellular carcinoma. Ann Surg Oncol 2015;22(Suppl 3):S1075-1082.

17. Wiesner R, Edwards E, Freeman R, et al. Model for End-Stage Liver Disease (MELD) and allocation of donor livers. Gastroenterology 2003;124:91-96.

18. Johnson PJ, Berhane S, Kagebayashi C, et al. Assessment of liver function in patients with hepatocellular carcinoma: a new evidence-based approachthe ALBI grade. J Clin Oncol 2015;33:550-558.

19. Sinn DH, Cho JY, Gwak GY, et al. Different survival of Barcelona clinic liver cancer stage $\mathrm{C}$ hepatocellular carcinoma patients by the extent of portal vein invasion and the type of extrahepatic spread. PloS One 2015;10:e0124434

20. Zhao Y, Cai G, Zhou L, et al. Transarterial chemoembolization in hepatocellular carcinoma with vascular invasion or extrahepatic metastasis: a systematic review. Asia Pac J Clin Oncol 2013;9:357-364
21. Yau T, Tang VY, Yao TJ, et al. Development of Hong Kong Liver Cancer staging system with treatment stratification for patients with hepatocellular carcinoma. Gastroenterology 2014;146:1691-1700.e3.

22. Omata M, Lesmana LA, Tateishi R, et al. Asian Pacific Association for the Study of the Liver consensus recommendations on hepatocellular carcinoma. Hepatol Int 2010;4:439-474.

23. Mazzaferro V, Sposito C, Bhoori S, et al. Yttrium-90 radioembolization for intermediate-advanced hepatocellular carcinoma: a phase 2 study. Hepatology 2013;57:1826-1837.

24. Wang JH, Kuo YH, Wang CC, et al. Surgical resection improves the survival of selected hepatocellular carcinoma patients in Barcelona clinic liver cancer stage C. Dig Liver Dis 2013;45:510-515.

25. Hasegawa K, Makuuchi M, Kokudo N, et al. Impact of histologically confirmed lymph node metastases on patient survival after surgical resection for hepatocellular carcinoma: report of a Japanese nationwide survey. Ann Surg 2014;259:166-170.

26. Kee KM, Wang JH, Lin CY, et al. Validation of the 7th edition TNM staging system for hepatocellular carcinoma: an analysis of 8,828 patients in a single medical center. Dig Dis Sci 2013;58:2721-2728.

27. Uka K, Aikata H, Takaki S, et al. Clinical features and prognosis of patients with extrahepatic metastases from hepatocellular carcinoma World J Gastroenterol 2007;13:414-420.

28. Okusaka T, Okada S, Ishii H, et al. Prognosis of hepatocellular carcinoma patients with extrahepatic metastases. Hepatogastroenterology 1997;44:251-257.

29. Jung SM, Jang JW, You CR, et al. Role of intrahepatic tumor control in the prognosis of patients with hepatocellular carcinoma and extrahepatic metastases. J Gastroenterol Hepatol 2012;27:684-689.

30. Uchino K, Tateishi R, Shiina S, et al. Hepatocellular carcinoma with extrahepatic metastasis: clinical features and prognostic factors. Cancer 2011;117:4475-4483.

31. Llovet JM, Ricci S, Mazzaferro V, et al. Sorafenib in advanced hepatocellular carcinoma. N Engl J Med 2008;359:378-390. 